\title{
A new and simple model for magneto-optics uncovers an unex- pected spin switching
}

\author{
G. P. Zhang ${ }^{1}$, Y. H. BAi ${ }^{2}$ and Thomas F. GeOrGE ${ }^{3}$
}

1' Department of Physics, Indiana State University, Terre Haute, IN 47809, USA

Office of Information Technology, Indiana State University, Terre Haute, IN 47809, USA

Office of the Chancellor and Center for Nanoscience

Departments of Chemistry $\&$ Biochemistry and Physics $\&$ Astronomy

University of Missouri-St. Louis, St. Louis, MO 63121, USA

PACS 78.20.Ls - Magneto-optical effects

PACS 75.78.Jp - Ultrafast magnetization dynamics and switching

PACS 75.40.Gb - Dynamic properties (dynamic susceptibility, spin waves, spin diffusion, dynamic scaling, etc.)

\begin{abstract}
In magneto-optics the spin angular momentum $S_{z}$ of a sample is indirectly probed by the rotation angle and ellipticity, which are mainly determined by the off-diagonal susceptibility $\chi_{x y}^{(1)}$. A direct and analytic relation between $S_{z}$ and $\chi_{x y}^{(1)}$ is necessary and of paramount importance to the success of magneto-optics, but is often difficult to acquire since quantum mechanically the relation is hidden in the sum-over-states. Here we propose a new and simple model to establish such a much needed relation. Our model is based on the Hookean model, but includes spin-orbit coupling. Under cw excitation, we show that $\chi_{x y}^{(1)}(\omega)$ is indeed directly proportional to $S_{z}$ for a fixed photon frequency $\omega$. Such an elegant relation is encouraging, and we wonder whether our model can describe spin dynamics as well. By allowing the spin to change dynamically, to our surprise, our model predicts that an ultrafast laser pulse can induce a spin precession; with appropriate parameters, the laser can even reverse spin from one direction to another. This works for both the circularly and linearly polarized light. The spin reversal window is narrow. These unexpected results closely resemble all-optical helicity-dependent magnetic switching found in much more complicated ferrimagnetic rare earth compounds. Therefore, we believe that our spin-orbit coupled model may find some important applications in spin switching processes, a hot topic in femtomagnetism.
\end{abstract}

Introduction. - Magneto-optical Faraday and Kerr - techniques are indispensable to modern magnetism investigations [1,2]. Such a technique is an ideal tool to investigate both static and dynamic evolutions of spin [3] , since it is a photon-in and photon-out technique and leaves the sample intact before and after the experimental measurement. The origin of the magneto-optics is rooted in the spin-orbit coupling and exchange interaction [7,8, where the rotational angle and ellipticity carry the information of the spin moment change. The classical understanding is developed through the harmonic oscillator model (Hookean model), augmented with the Lorentz force term [7, 8,

$$
-\frac{d^{2} \mathbf{r}}{d t^{2}}+2 \gamma \mathbf{v}+\Omega^{2} \mathbf{r}=\frac{q \mathbf{E}(t)}{m}+q \mathbf{B} \times \mathbf{v}
$$

where $\mathbf{r}$ and $\mathbf{v}$ are the position and velocity of the electron, $\gamma$ is the damping, $\Omega$ is the resonance frequency of the harmonic oscillator, $q$ is the electron charge, $\mathbf{B}$ is the external magnetic field, and $\mathbf{E}(t)$ is the electric field of light. The second term on the right-hand side is the Lorentz force term and is the only term that is responsible for the magneto-optical effect.

Recently, Hinschberger and Hervieux 9] extended the harmonic oscillator model by introducing more higher order terms, so the nonlinear optical response can be computed as well. The spin-orbit interaction is included through the current operator, which becomes another source term in the Maxwell equation. The spin contribution is lumped into a single parameter that represents the modification due to the spin-orbit interaction. Very 
recently, the same model was used to develop a quantum theory for the inverse Faraday effect [10.

Despite the great success of the harmonic oscillator model to describe the magneto-optics, the link between the magnetic spin moment and the magneto-optical signal can not be easily established from Eq. (11) since the harmonic oscillator model contains no spin. Argyres 7 provided a hand-waving argument from the summation over the magnetic electrons by recognizing

$$
\begin{aligned}
\int_{v} d \mathbf{k} & =(2 \pi)^{3} \times \text { no. of magnetic electrons per volume } \\
& =(2 \pi)^{3} \frac{M_{s}}{\frac{1}{2} g \mu_{B}},
\end{aligned}
$$

where $\mathbf{k}$ is the crystal momentum, $M_{S}$ is the saturation magnetization, $g$ is the Landé $g$-factor, and $\mu_{B}$ is the Bohr magneton. Even in modern magneto-optics bandstructure theory, such a relation between the spin moment and the off-diagonal susceptibility is also hidden in the summation over the crystal momentum [11. An alternative is to adopt an relaxation-time approximation 8]. Given the importance of the time-resolved magnetooptical response in laser-induced ultrafast spin dynamics [3], this is highly unsatisfactory in comparison with linear optics, where the susceptibility can be directly connected to the dipole transition [12,13.

In this paper, we make a moderate attempt to establish an analytic link between the spin angular momentum and off-diagonal susceptibility in the classical Hookean model. Our treatment is simple and straightforward, and physically more intuitive. We first replace the above Lorentz term by a spin-orbit coupling term. Then, we use the equation of motion method to find the evolution of the electron position. Under the continuous wave (cw) excitation, we obtain an analytic expression for the off-diagonal susceptibility $\chi_{x y}^{(1)}$. We find that $\chi_{x y}^{(1)}$ is indeed directly proportional to the product of the spin $S_{z}$ and the spinorbit coupling $\lambda$. Different from the regular linear optical response, the susceptibility has four poles, instead of two. These additional poles result from the spin-orbit coupling. Encouraged by this elegant result, we are curious whether such a simple model could accommodate spin precession. To our surprise, by allowing spin momentum to change dynamically with time, we find that this spin-orbit coupled Hookean model is capable of describing laser-induced ultrafast spin precession as well. For most laser parameters, our model predicts a spin canting, but in a narrow parameter range, we find spin switching. This largely unexpected spin switching has the same character as observed in bulk ferrimagnets, where both linearly and circularly polarized light can switch spins, and both the laser duration and field amplitude have a strong effect on the spin switching. This model might have some important applications for all-optical helicity-dependent magnetic switching [14-16].

The rest of the paper is arranged as follows. Section II is devoted to the theoretical formalism, where the an- alytic results for the diagonal and off-diagonal susceptibilities and the equations of motion for position, momentum, spin and orbital angular momenta are shown. In Sec. III, we report the spin switching in our spin-orbit coupled Hookean model. The conclusion is provided in Sec. IV.

Theoretical formalism. - Interest in time-resolved magneto-optics is fueled by the discovery of the laserinduced ultrafast spin dynamics in ferromagnets [3, 6. The field has grown enormously over two decades [5, 17, with the discovery of all-optical helicity-dependent magnetic switching 14 16, 18. Our initial motivation for this study came from a long discussion in 1999 with one of our experimental colleagues [19] who suggested whether a similar harmonic oscillator model as in traditional nonlinear optics 12 could be developed. However, it was unclear then whether a model, even if developed, could contain enough physics to be useful to experimentalists. New investigations have now demonstrated the potential value in this direction 9, 10. One big issue, however, is how to include the spin-orbit coupling (SOC) quantum mechanically. In 2011, when we investigated ultrafast spin linear reversal 20], we found a possibility to include SOC within the Heisenberg model, but when we tried to include the laser field, a major difficulty appeared: How to couple the laser field to the system in the Heisenberg model? In particular, the vector potential of the laser field is spin-independent. In 2014, while discussing with our colleagues from University of Colorado 21, we realized that the coupling could also be treated through the harmonic oscillator model. The only thing left to be established is the connection among momentum, spin and orbital angular momenta. In 1998, to explain how SOC rotates the polarization plane of linearly polarized light, Hübner 22] noticed that an additional force from SOC acts upon the electron through $i \hbar \dot{\mathbf{p}}=\left[H_{\text {soc }}, \mathbf{p}\right]=$ force. We wondered whether a similar equation can be derived for the spin and orbital as well.

However, while testing this idea, it became immediately clear to us that we ought to replace the Lorentz term in the original harmonic oscillator model by SOC. Thus our Hamiltonian takes the form [20, 23, 26.

$$
H=\frac{\mathbf{p}^{2}}{2 m}+\frac{1}{2} m \Omega^{2} \mathbf{r}^{2}+\lambda \mathbf{L} \cdot \mathbf{S}-e \mathbf{E}(t) \cdot \mathbf{r}
$$

where the first term is the kinetic energy operator of the electron, the second term is the harmonic potential energy operator with system frequency $\Omega, \lambda$ is the spin-orbit coupling in units of $\mathrm{eV} / \hbar^{2}, \mathbf{L}$ and $\mathbf{S}$ are the orbital and spin angular momenta in units of $\hbar$, respectively, and $\mathbf{p}$ and $\mathbf{r}$ are the momentum and position operators of the electron, respectively.

To start with, we treat all the operators quantum mechanically and use the Heisenberg equation of motion 22 to find four coupled equations for the position $\mathbf{r}$, momentum $\mathbf{p}$, spin $\mathbf{S}$ and orbital $\mathbf{L}$ angular momenta, respec- 
tively:

$$
\begin{aligned}
\frac{d \mathbf{r}}{d t} & =\frac{\mathbf{p}}{m}-\lambda(\mathbf{r} \times \mathbf{S}), \\
\frac{d \mathbf{p}}{d t} & =-m \Omega^{2} \mathbf{r}+e \mathbf{E}(t)-\lambda \mathbf{p} \times \mathbf{S}, \\
\frac{d \mathbf{S}}{d t} & =\lambda(\mathbf{L} \times \mathbf{S}), \\
\frac{d \mathbf{L}}{d t} & =-e \mathbf{E}(t) \times \mathbf{r}-\lambda(\mathbf{L} \times \mathbf{S}) .
\end{aligned}
$$

Up to this step, these equations are exact. Next we make the Hartree-Fock approximation by replacing the operators with their respective expectation values; for simplicity we keep the same symbols. This allows us to solve these coupled equations analytically.

These four coupled equations contain incredibly rich physics. First we note that the position's change with time is renormalized by the spin-orbit coupling (see Eq. (41)). This is very different from the conventional equation of motion for the electron under an external magnetic field B, where B enters the equation through the momentum alone (see Eq. (10). The momentum change also contains a contribution from SOC. Second, the spin does not depend on the laser field directly, but instead on the orbital momentum. Upon the laser excitation, the orbital angular momentum $\mathbf{L}$ is first excited (Eq. (7)). The spin dynamics is driven by the the spin-orbit coupling through a spin-orbit torque (Eq. (6) ). The process is similar to a recent study in a magnetic semiconductor by Lingos and coworkers 27] and in a more complicated magnetic system 28. The module of spin is conserved, or $\dot{\mathbf{S}} \cdot \mathbf{S}=0$, as seen from Eq. (6).

Since we are interested in an analytic solution, we consider a case with constant spin. We take the derivative of Eq. (4) with respect to time and then substitute Eq. (5) into it. After some rearrangement, the equation of motion for the electron position can be simplified to a single equation,

$$
\ddot{\mathbf{r}}+2 \lambda \dot{\mathbf{r}} \times \mathbf{S}+\left(\Omega^{2}-\lambda^{2} S^{2}\right) \mathbf{r}-\lambda^{2}(\mathbf{r} \cdot \mathbf{S}) \mathbf{S}=\frac{e \mathbf{E}(t)}{m} .
$$

This resultant equation closely resembles the classical Hookean harmonic oscillator equation (Eq. (1)), but with some differences. The second term on the left-hand side is not a damping term; instead, it represents a rotation along an orthogonal direction. The harmonic potential term (third term on the left-hand side) is modified with a frequency red-shifted to $\left(\Omega^{2}-\lambda^{2} S^{2}\right)$, where $S$ is the module of $\mathbf{S}$. We align our spin along the $z$ axis or $\mathbf{S}=S_{z} \hat{z}$. We apply a cw optical field polarized along the $x$ direction, $E_{x}(t)=A_{x} e^{i \omega t}+c c$., where $A_{x}$ is the amplitude and $\omega$ is the laser frequency. From Eq. (8), we find two coupled equations for $x$ and $y$ as

$$
\begin{aligned}
\ddot{x}+2 \lambda S_{z} \dot{y}+\left(\Omega^{2}-\lambda^{2} S_{z}^{2}\right) x & =\frac{e E_{x}(t)}{m} \\
\ddot{y}-2 \lambda S_{z} \dot{x}+\left(\Omega^{2}-\lambda^{2} S_{z}^{2}\right) y & =0 .
\end{aligned}
$$

To find a stationary solution, we assume $x(t)=x_{0} \mathrm{e}^{i \omega t}$ and $y(t)=y_{0} \mathrm{e}^{i \omega t}$. We substitute them back into Eqs. (7) and (8) to find

$$
\begin{aligned}
& x_{0}=\frac{e}{m} \frac{D}{D^{2}-C^{2}} A_{x} \\
& y_{0}=\frac{i e}{m} \frac{C}{D^{2}-C^{2}} A_{x},
\end{aligned}
$$

where $D=\Omega^{2}-\omega^{2}-\lambda^{2} S_{z}^{2}$ and $C=2 \lambda S_{z} \omega$.

To compute the susceptibility, we need to find the polarizations along the $x$ and $y$ directions [13: $P_{x}(\omega)=-N e x_{0}$ and $P_{y}(\omega)=-N e y_{0}$. The diagonal susceptibility (in SI units) is just $\chi_{x x}^{(1)}=P_{x} / A_{x} \epsilon_{0}$, and the off-diagonal susceptibility (in SI units) is $\chi_{x y}^{(1)}=P_{y} / A_{x} \epsilon_{0}$, i.e.,

$$
\begin{aligned}
\chi_{x x}^{(1)}(\omega) & =-\frac{N e^{2}}{\epsilon_{0} m} \frac{\Omega^{2}-\omega^{2}-\lambda^{2} S_{z}^{2}}{\left(\Omega^{2}-\omega^{2}-\lambda^{2} S_{z}^{2}\right)^{2}-\left(2 \lambda S_{z} \omega\right)^{2}}(13) \\
\chi_{x y}^{(1)}(\omega) & =-i \frac{N e^{2}}{\epsilon_{0} m} \frac{2 \lambda S_{z} \omega}{\left(\Omega^{2}-\omega^{2}-\lambda^{2} S_{z}^{2}\right)^{2}-\left(2 \lambda S_{z} \omega\right)^{2}}(14)
\end{aligned}
$$

where $N$ is the number density and $\epsilon_{0}$ is the permittivity in the vacuum. These two equations are insightful. Equation (13) shows that SOC creates two additional poles on the frequency axis at $\omega= \pm\left(\Omega-\lambda S_{z}\right)$ and $\omega= \pm\left(\Omega+\lambda S_{z}\right)$. Without SOC, we recover the well-known linear susceptibility results 13 . Second, the off-diagonal linear susceptibility $\chi_{x y}^{(1)}$ is indeed proportional to the spin angular momentum and spin-orbit coupling. To the best of our knowledge, such an analytic expression has never been found before. It is truly gratifying that our simple model can reproduce such an elegant relation and sets up a classical analogue to the linear optics counterpart [11,13], placing the entire magneto-optical theory on a firmer ground for the first time [11.

Surprising spin switching. - Being able to analytically connect the off-diagonal susceptibility to the spin angular momentum is encouraging, but the spin angular momentum is assumed to be static, though we have four coupled equations. We are curious as to what happens if we allow the spin to dynamically change with time under a laser pulse.

We choose laser pulses of two different kinds. For a linearly polarized $(\pi)$ pulse, the electric field is $\mathbf{E}(t)=$ $A_{0} \mathrm{e}^{-t^{2} / \tau^{2}} \cos (\omega t) \hat{x}$, where $\omega$ is the laser carrier frequency, $\tau$ is the laser pulse duration, $A_{0}$ is the laser field amplitude, $t$ is time, and $\hat{x}$ is the unit vector along the $x$ axis. Note that the results are the same if the field is along the $y$ axis. The electric field for the right and left circularly polarized pulses $\left(\sigma^{+}\right.$and $\left.\sigma^{-}\right)$is $\mathbf{E}(t)=$ $A_{0} \mathrm{e}^{-t^{2} / \tau^{2}}( \pm \sin (\omega t) \hat{x}+\cos (\omega t) \hat{y})$, where $+(-)$ refers to $\sigma^{+}\left(\sigma^{-}\right)$. We then compute the spin evolution by numerically solving the four coupled equations (447).

We find that for most sets of laser parameters the spin precesses with time, but for a few special set of laser parameters, the laser can directly switch spin, without 
the presence of a magnetic field. This is a big surprise to us. Figure 1(a) shows that a left-circularly polarized pulse $\left(\sigma^{-}\right)$of duration $\tau=60$ fs and field amplitude $A_{0}=0.035 \mathrm{~V} / \AA$ can switch the spin, initialized along the $-z$ axis with $S_{z}(0)=-2.2 \hbar$, to $2 \hbar$. We choose the spinorbit coupling $\lambda=0.06 \mathrm{eV} / \hbar^{2}$, and $\hbar \omega=\hbar \Omega=1.6 \mathrm{eV}$. Note that our excitation is slightly off-resonant since new poles come from SOC (see Eq. (14)). We find that upon the laser excitation, the spin first precesses strongly without any oscillation toward the $x y$ plane and the exact precession of $S_{x}$ and $S_{y}$ depends on the initial phase of the laser pulse, but the precession of $S_{z}$ is always the same. The spin reaches the negative maximum at $34 \mathrm{fs}$, exactly when $S_{z}$ passes through zero. $S_{z}$ is successfully switches to $2 \hbar$ at $80 \mathrm{fs}$, where the spin rotates $155.9^{\circ}$. Such a spin switching is remarkable, and to the best of our knowledge, has never been reported before. Will this work for a $\sigma^{+}$ pulse?

Figure1(b) shows that both $\sigma^{+}$and $\sigma^{-}$can switch spin within a few hundred femtoseconds. Our results reveal a stringent symmetry constraint on the spin switching: The $\sigma^{-}$light only switches the spin from down to up, while the $\sigma^{+}$light switches the spin from up to down, not the other way around. Numerically we find that $\sigma^{+}$ only slightly perturbs the down spin, because the phase mismatch between the position of the electron and the laser field imposes a negative torque on the spin switching. We also try to use linearly polarized light $(\pi)$. Figure 1(c) shows that for the same amplitude of $0.035 \mathrm{~V} / \AA$, the $\pi$ pulse can not switch the spin, and only a small spin change is noticed. To induce spin switching, we need to increase $A_{0}$ above $0.2 \mathrm{~V} / \AA$, or 5.7 times higher than used for either $\sigma^{+}$or $\sigma^{-}$.

Our finding reminds us some of familiar experimental results found in all-optical helicity-dependent magnetic switching in ferrimagnetic rare-earth bulk materials 15. 16. For instance, experimentally, Stanciu et al. [14] showed that both $\sigma^{+}$or $\sigma^{-}$can switch spins; and Alebrand et al. 29 found that a $\sigma$ pulse appears more powerful than a $\pi$ pulse. In addition, Vahaplar et al. 30, found that the actual spin reversal window of the laser fluence is very narrow and asymmetric (see Fig. 1(d)). We wonder whether a similar switch window exists for our system. Figure 1(e) shows that as $A_{0}$ increases, the final spin $S_{z}$ first increases sharply (see the empty circles) and then reaches its maximum of $2 \hbar$ at $A_{0}=0.035 \mathrm{~V} / \AA$, where the spin is reversed. If we increase the field amplitude further, $S_{z}$ decreases and eventually the spin switching disappears. The reversal window is indeed very narrow and asymmetric (see the shaded region in Fig. 1(b)), only from 0.026 to $0.042 \mathrm{~V} / \AA$.

To understand how such a narrow reversal window is formed, we systematically monitor the orbital and spin angular momentum changes as a function of time. We find that while the orbital angular momentum always increases with the field amplitude, the spin change is nontrivial. If the laser is too weak, weaker than $0.026 \mathrm{~V} / \AA$, the spin either does not rotate at all or simply cants to the $-x$ axis, without switching. If $A_{0}$ is too strong, between $0.035 \mathrm{~V} / \AA$ and $0.06 \mathrm{~V} / \AA$, the final spin overshoots and cants to the $+y$ axis. It is this balance between these two limits that leads to the narrow reversal window. Figure 1(f) shows the detailed dependence of the final spin components as a function of amplitude. Note that the actual dependence at the higher amplitude is very complicated, and further spin switching and canting are possible. If we shorten the pulse duration, we find that the switching window is shifted to the high intensity end and is slightly widened (see Fig. 1(e)). This shift is expected since to switch the spin, the laser field must transfer enough energy and angular momentum to the system. If the pulse is too short, the spin does not have enough time to reverse before the laser pulse is gone.

Up to now, all the results are obtained with a fixed $\lambda$ and $\hbar \omega$. Next we tune them separately. We first fix $\lambda$ at $0.06 \mathrm{eV} / \hbar^{2}$. Figure 2(a) shows that the energy difference $(\Delta E=\hbar \Omega-\hbar \omega)$ between the laser photon energy and the system energy affects the spin reversal window strongly. We increase $\Delta E$ from -0.05 to $0.05 \mathrm{eV}$ in steps of 0.01 $\mathrm{eV}$. We see that at $\Delta E=-0.05 \mathrm{eV}$, the spin reversal window is broader but there is no spin reversal. To see this clearly, we choose a point with an optimal field amplitude (see the small dashed box in Fig 2(a)), and plot the spin angular momentum as a function of time in Fig 2(b) (see the dashed line). We notice that the spin only oscillates between two extremes (see the dashed line in Fig 2(b)), and does not reverse even at this optimal laser amplitude. However, as we increase $\Delta E$ further close to $0 \mathrm{eV}$, the spin reversal window sharpens, with the peaks on the high end of the laser field amplitude dropping off, and spin reversal starts. Our result at $\Delta E=0 \mathrm{eV}$ is highlighted in red. Above $\Delta E=0 \mathrm{eV}$, the reversal window broadens and forms a plateau. This means that the spin reversal can occur at different $A_{0}$. We also choose a point at $\Delta E=$ $0.05 \mathrm{eV}$ (see the solid box), and plot the spin change in Fig. 2(b) (solid line), where we see that the spin is indeed reversed, but with a small ringing.

Next we fix $\Delta E$ at $0 \mathrm{eV}$, and increase $\lambda$ from 0.01 to $\lambda=0.08 \mathrm{eV} / \hbar^{2}$, in steps of $0.01 \mathrm{eV} / \hbar^{2}$. Figure 2(c) shows that at $\lambda=0.01 \mathrm{eV} / \hbar^{2}$, even with an optimal amplitude (see the dashed box in Fig. 2(c)), the spin only oscillates and does not reverse (see the dashed line in Fig. 2(d)). As $\lambda$ increases, the spin reversal window also changes its shape, and the optimal field amplitude gradually shifts to a high value and then converges. A minimum $\lambda_{\text {min }}$ is required to reverse the spin. We estimate that the minimum $\lambda_{\text {min }}$ to reverse spin is around $\lambda=0.03 \mathrm{eV} / \hbar^{2}$ for our current parameters. Figure 2(d) also shows a successful spin reversal for our largest $\lambda=0.08 \mathrm{eV} / \hbar^{2}$ (see the solid line). The entire process is similar to our results at $\lambda=0.06 \mathrm{eV} / \hbar^{2}$. At present, we are still investigating the possibility to develop a simple physics picture from Eqs. (4)-(7).

So many similarities in the spin reversal between our spin-orbit coupled harmonic oscillator and ferrimagnetic 
rare earth bulks are astonishing and highly unexpected. We wonder whether they share the common origin. Physically, they both have the spin-orbit coupling, are coupled with the laser field, and do not rely on an external magnetic field. One apparent difference between them is that in our system the spin reversal is much faster with the presence of the laser pulse, but in ferrimagnets on the order of several picoseconds when the laser field is gone. However, this difference may be explained by the size effect. Our system contains one single site; regular ferrimagnets have a lot more. It could be that in those ferrimagnets, the spin reversal initially occurs in the small ranges with the strongest laser field amplitude, and the propagation of the spin reversal, from those initial excited sites to the remote sites, takes several picoseconds [18. Nevertheless, we caution that these ferrimagnets consist of two sublattices and are quite different from our model. Additional research is needed in this direction to completely illuminate the true mechanism.

Conclusion. - We have proposed a new and simple spin-orbit coupled harmonic oscillator model for magnetooptics to establish the analytic relation between the experimentally accessible off-diagonal susceptibility $\chi_{x y}^{(1)}(\omega)$ and experimentally inaccessible spin angular momentum $S_{z}$. We show that under cw excitation, for the same photon frequency $\omega, \chi_{x y}^{(1)}(\omega)$ is directly proportional to the product of the spin-orbit coupling $(\lambda)$ and the spin moment $\left(S_{z}\right)$, or $\lambda S_{z}$. Different from the traditional optical response, the off-diagonal susceptibility has four poles on the frequency axis, instead of two. These two extra poles are a result of spin-orbit coupling. Therefore, our results provide an important theoretical foundation for magnetooptics, in contrast to the prior efforts, where such a relation is obtained approximately or hidden in the sumover-states. To our surprise, our model, once the spin is allowed to change and under the laser excitation, predicts the laser-induced ultrafast spin precession; with appropriate laser parameters, the spin reverses from one direction to another. This works for both the circularly and linearly polarized light, but the former appears more powerful than the latter. The spin reversal window is very narrow due to the stringent requirement on the precession of the spin. All these results are similar to the all-optical helicity-dependent magnetic switching (AOS) observed in ferrimagnets. At present, it is unknown whether the same physics plays the role in both systems. We believe that an extension of our current model to a large system could be fruitful to AOS, where the research has been very intensive, with the possible applications in ultrafast magnetic storage as demonstrated in several latest investigations [15, 16.

$$
* * *
$$

This work was solely supported by the U.S. Department of Energy under Contract No. DE-FG02-06ER46304.
Part of the work was done on Indiana State University's quantum cluster and high-performance computers. The research used resources of the National Energy Research Scientific Computing Center, which is supported by the Office of Science of the U.S. Department of Energy under Contract No. DE-AC02-05CH11231. This work was performed, in part, at the Center for Integrated Nanotechnologies, an Office of Science User Facility operated for the U.S. Department of Energy (DOE) Office of Science by Los Alamos National Laboratory (Contract DE-AC5206NA25396) and Sandia National Laboratories (Contract DE-AC04-94AL85000).

\section{REFERENCES}

[1] M. H. Kryder, J. Appl. Phys. 57, 3913 (1985).

[2] A. Vaterlaus, T. Beutler, and F. Meier, Phys. Rev. Lett. 67, 3314 (1991); P. L. Scott and C. D. Jeffries, Phys. Rev. 127, 32 (1962).

[3] E. Beaurepaire, J.C. Merle, A. Daunois, and J.-Y. Bigot, Phys. Rev. Lett. 76, 4250 (1996).

[4] L. Guidoni, E. Beaurepaire, and J.-Y. Bigot, Phys. Rev. Lett. 89, 017401 (2002).

[5] J.-Y. Bigot, W. Hübner, Th. Rasing, and R. Chantrell, Eds. Ultrafast Magnetism I, Springer Proceedings in Physics 159 (2015).

[6] G. P. Zhang and W. Hübner, Phys. Rev. Lett. 85, 3025 (2000).

[7] P. N. Argyres, Phys. Rev. 97, 334 (1955).

[8] J. L. Erskine and E. A. Stern, Phys. Rev. B 8, 1239 (1973).

[9] Y. Hinschberger and P.-A. Hervieux Phys. Rev. B 88, 134413 (2013)

[10] M. Battiato, G. Barbalinardo, and P. M. Oppeneer, Phys. Rev. B 89, 014413 (2014).

[11] G. P. Zhang, W. Hübner, G. Lefkidis, Y. Bai, and T. F. George, Nature Phys. 5, 499 (2009).

[12] N. Bloembergen, Nonlinear Optics, 4th Ed. (World Scientific, Singapore, 1996)

[13] R. W. Boyd, Nonlinear Optics (Academic Press, San Diego, 1992), p. 25.

[14] C. D. Stanciu, F. Hansteen, A. V. Kimel, A. Kirilyuk, A. Tsukamoto, A. Itoh, and Th. Rasing, Phys. Rev. Lett. 99, 047601 (2007).

[15] S. Mangin et al., Nature Mater. 13, 286 (2014).

[16] C.-H. Lambert et al., Science 345, 1337 (2014).

[17] A. Kirilyuk, A. V. Kimel, and Th. Rasing, Rev. Mod. Phys. 82, 2731 (2010).

[18] T. A. Ostler et al., Nat. Commun. 3, 666 (2012).

[19] J.-Y. Bigot, private communication.

[20] G. P. Zhang, J. Phys.: Condens. Mat. 23, 206005 (2011).

[21] Patrik Grychtol, private communication.

[22] W. Hübner, in Nonlinear Optics in Metals, ed. by K. H. Bennemann (Clarendon Press, Oxford, 1998), p. 268.

[23] M. S. Si and G. P. Zhang, J. Phys.: Condens. Mat. 22, 076005 (2010).

[24] G. P. Zhang and T. F. George, J. Phys.: Condens. Mat. 25, 366002 (2013).

[25] G. P. Zhang, M. Q. Gu, and X. S. Wu, J. Phys.: Condens. Mat. 26, 376001 (2014). 

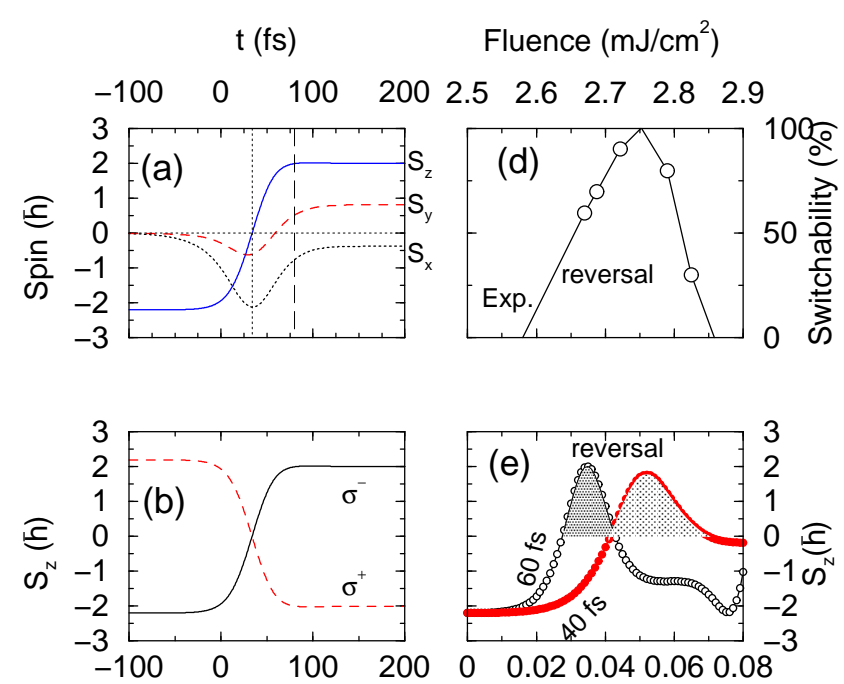

$\mathrm{A}_{0}(\mathrm{~V} / \mathrm{A})$
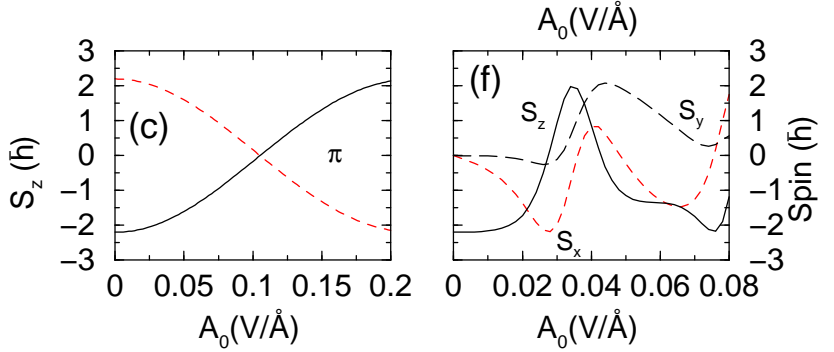

Fig. 1: (a) All-optical spin reversal for $S_{x}, S_{y}$ and $S_{z}$ as a function of time $t$. The vertical dotted line denotes the time when $S_{z}$ passes through zero, and the vertical dashed line denotes the time when the spin reversal starts. Here $\sigma^{-}$is used, the laser pulse duration is $\tau=60 \mathrm{fs}$, and the field amplitude is $0.035 \mathrm{~V} / \AA$. (b) The $\sigma^{-}$pulse (solid line) only switches spin from down to up, while the $\sigma^{+}$pulse (dashed line) only switches spin from up to down. (c) The $\pi$ pulse can switch spin from up to down or from down to up, but at a much higher field amplitude. (d) Experimental spin reversal window from Ref. 28. (e) Final spin angular momentum $S_{z}$ as a function of the laser field amplitude for laser durations $\tau=60$ fs (empty circles) and $40 \mathrm{fs}$ (filled circles). The shaded regions are the spin reversal window. (f) As the field amplitude increases, the spin angular momentum changes from non-switching, canting along the $-x$ axis, switching, and canting along the $+y$ axis.

[26] G. P. Zhang, M. S. Si, Y. H. Bai, and T. F. George, J. Phys.: Condens. Mat. 27, 206003 (2015).

[27] P. C. Lingos, J. Wang, and I. E. Perakis, Phys. Rev. B 91, 195203 (2015).

[28] T. Li, A. Patz, L. Mouchliadis, J. Yan, T. A. Lograsso, I. E. Perakis, and J. Wang, Nature 496, 69 (2013).

[29] S. Alebrand, A. Hassdenteufel, D. Steil, M. Cinchetti, and M. Aeschlimann, Phys. Rev. B 85, 092401 (2012).

[30] K. Vahaplar et al., Phys. Rev. B 85, 104402 (2012).

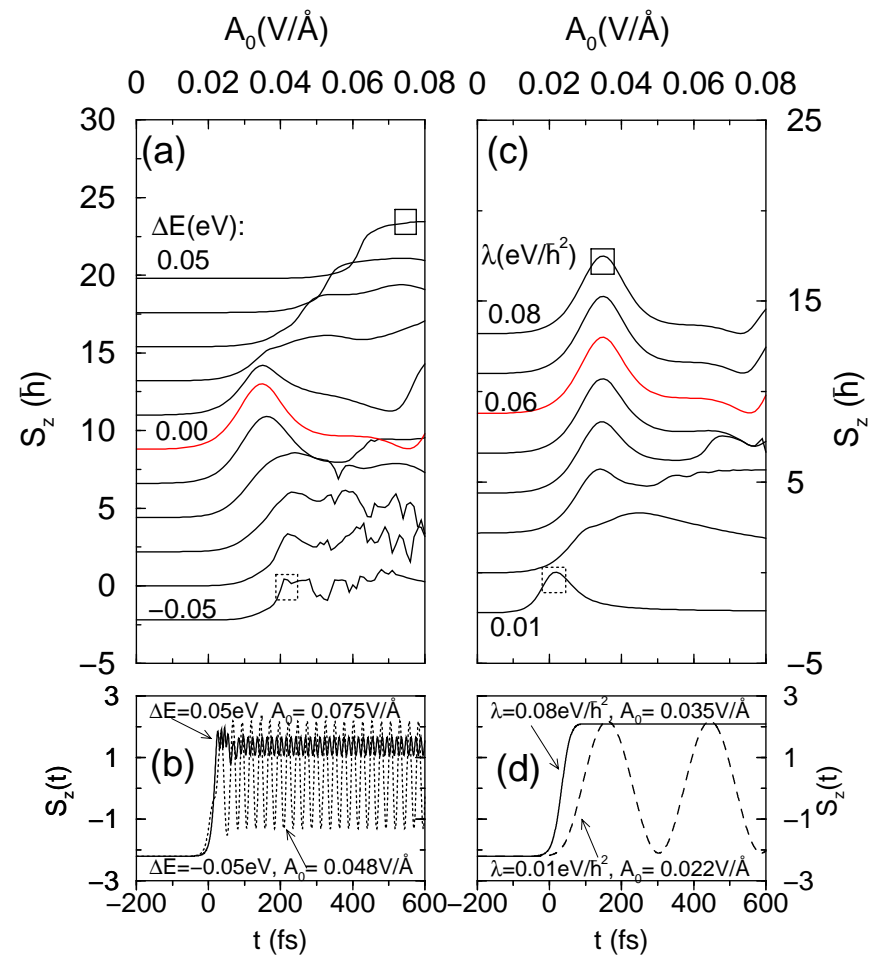

Fig. 2: (a) Dependence of the spin reversal window on the energy difference $\Delta E$ with fixed $\lambda=0.06 \mathrm{eV} / \hbar^{2}$, where $\Delta E=$ $\hbar \omega-\hbar \Omega$. $\Delta E$ increases from -0.05 to $0.05 \mathrm{eV}$ in steps of $0.01 \mathrm{eV}$ from the bottom to top. All the curves are shifted vertically for clarity. The solid and dashed boxes denote two representative cases, whose spin change is shown in (b). (b) Spin angular momentum change with time for the laser field amplitude of $A_{0}=0.075 \mathrm{~V} / \AA$ and $\Delta E=0.05 \mathrm{eV}$ (solid line) and $A_{0}=$ $0.049 \mathrm{~V} / \AA$ and $\Delta E=-0.05 \mathrm{eV}$ (dashed line). (c) Dependence of the spin reversal window on the spin-orbit coupling $\lambda$, with $\Delta E$ fixed at $0 \mathrm{eV}$. Here $\lambda$ increases from 0.01 to $0.08 \mathrm{eV} / \hbar^{2}$ in steps of $0.01 \mathrm{eV} / \hbar^{2}$. (d) Spin angular momentum change with time for $A_{0}=0.035 \mathrm{~V} / \AA$ and $\lambda=0.08 \mathrm{eV} / \hbar^{2}$ (solid line) and $A_{0}=0.022 \mathrm{~V} / \AA$ and $\lambda=0.01 \mathrm{eV} / \hbar^{2}$ (dashed line). 